\title{
THE REFERENCE OF CARDIAC PAIN TO A PHANTOM LEFT ARM
}

\author{
BY \\ HENRY COHEN AND H. WALLACE JONES \\ From the Royal Infirmary, Liverpool \\ Received September 16, 1942
}

Two cases in which anginal pain was referred in part to a "phantom " left arm are here recorded as a contribution to the problem of the mechanism of referred pain. In both, (1) the left arm had been amputated more than 25 years before the onset of cardiac pain, (2) there was evidence, both clinical and cardiographic, of gross myocardial damage, and (3) the "phantom" component of the pain was abolished, or significantly modified, by anæsthetizing the left brachial plexus.

Case I. Cardiac infarction; left bundle branch block. Phantom left arm since 1916 following amputation through upper arm; cardiac pain referred to phantom and chest (April, 1942). Left brachial plexus block eliminated "phantom" component.

H. T. (admitted 26/5/42), male, aged 45 years, in charge of social department of Tramways Depôt for 16 years; married; childless; wife healthy. Smokes 10 to 15 cigarettes daily but life-long teetotaler. No history of illness before 1916, and except for occasional colds and winter cough excellent health since discharge from the army. No significant family history of cardiovascular sclerosis.

On October 8, 1916, in France, he was wounded above the left elbow; ten days later the arm was amputated because of gangrene. Progress was good though three months later, owing to a persistent slight discharge, he had another operation. The wound then healed rapidly and completely. From the time of the amputation he has had a phantom arm, at first equal in length to his right arm, though gradually it has shortened to about half its original length; it moves with the shoulder but the elbow, forearm and hand remain fixed-the elbow at about $90^{\circ}$ flexion, the forearm in mid-pronation-supination, and hand and finger joints slightly flexed-“" as if clutching a ball." The hand dominates the phantom and he can discern all fingers clearly. The awareness of the phantom is not due to pain but to a tingling which he is usually able to ignore; "you get quite used to its being there." Wet weather intensifies the tingling.

On April 6, 1942, an hour after rising, he was seized suddenly with pain in the substernal and left submammary region which spread to the stump and radiated down the phantom to the fingers, though the worst pain was felt in the phantom biceps region. Waterbrash and one small vomit accompanied this attack, which passed off completely in less than an hour; he went to his work that day and experienced no further pain, breathlessness, or other chest symptoms. During the following six days he had occasional twinges of the

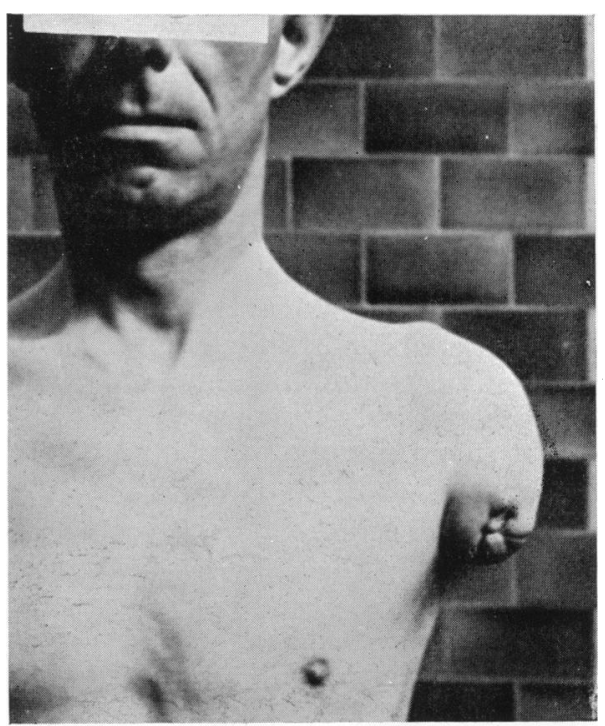

FIG. 1.-Case 1. Showing the amputation stump and scar.

pain, but on April 12, whilst in bed, he had an attack similar in type, intensity, and duration to that of April 6. Since then the pain has recurred on undue effort, though the effort needed to produce pain radiating to the phantom varies; rest for a minute or two invariably eases the pain, which is induced by less effort in the morning than in the afternoon.

Examination showed a stump, four inches long (Fig. 1), with free and full movement at the shoulder joint and slight hyperæsthesia of the scar.

The heart was slightly enlarged and Dr. Roberts reported: Slight enlargement of left ventricle; 
no evidence of pathological calcification, or of pulmonary lesion. The sounds were normal, the rhythm regular, and there was no murmur. B.P. 110/60. Pulse rate 78. Early locomotor brachial artery and slight retinal arteriosclerosis. There was typical left bundle branch block (Fig. 4A).

There were no significant clinical changes in the lungs, nervous system, or abdomen; no evidence of sepsis in mouth (edentulous) or naso-pharynx, and no pyrexia during his stay in hospital; no thyroid change, no skin rash, nor palpable lymph nodes. The urine was normal except for a few squamous cells and leucocytes with large numbers of calcium oxalate crystals. A blood count was also normal. The Wassermann reaction was negative.

On June 5, 1942, Mr. Edwards blocked the left brachial plexus with 60 c.c. of 1 per cent solution of procaine and Fig. 2 shows the extent of the resulting anæsthesia. No ocular or vascular changes
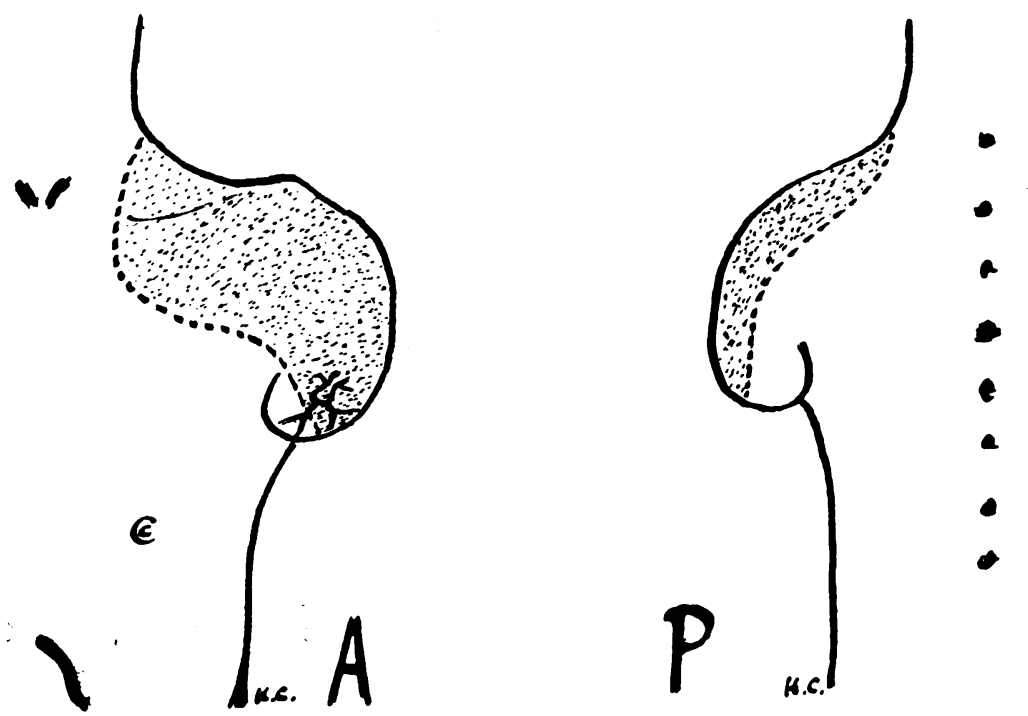

FIG. 2.-Case 1. The dotted area maps the anæsthesia resulting from the brachial plexus block. A. Anterior view. P. Posterior view.

were seen. The lower part of the scar remained sensitive though the patient felt that the whole phantom was numbed. Ten minutes later he walked 400 yards briskly; pain was felt in the chest only; the arm was quite free from pain though he had never previously experienced pain in the chest without radiation to the arm. When the anæsthesia passed off, exercise brought on pain in both chest and arm.

The variability in the intensity of effort required to produce the pain prevented quantitive observations on this patient. It is, however, of interest to note that since the brachial plexus block the phantom has shortened; he is no longer aware of arm or forearm, the hand appearing to be attached directly to the stump, and exertion now causes pain to appear in the chest and hand and/or stump simultaneously.

Case II. Angina of effort; coronary arteriosclerosis. Phantom left forelimb since 1916 following disarticulation through shoulder joint; cardiac pain present and gradually intensifying for 5 weeks; referred to phantom, neck, jaws, and chest. Left brachial plexus block practically eliminated phantom component, and modified sequence of appearance of pain in different sites.

J. T. (admitted 26/5/42), male, aged 51 years, a credit draper with no strenuous work; single; smokes 25 to 30 cigarettes daily and drinks an occasional beer. Father died at 52 from angina pectoris, mother at 74 from cerebral hæmorrhage, 5 sibs alive and well, a sister died aged 9 of spinal caries.

On August 9, 1916, his left humerus was shattered by machine-gun fire. He was treated at a field dressing station, but two days later, because of gangrene, the arm was disarticulated through the shoulder joint. A painful phantom appeared immediately, confined to the left hand, which was somewhat nearer to the shoulder than the right hand, and hanging at his side with fist clenched and nails digging into the palm, though the individual fingers were not distinguishable. The hand felt constantly swollen and burning, worse by night, and there were sharp stabbing pains on excitement. No voluntary movement of the hand or fingers was possible, but during the first two months he noticed that when he gripped with his right hand the phantom hand seemed to clench more tightly. 
In 1917, an attack of acute appendicitis with operation caused an exacerbation of pain which subsided in a month. During the next 10 years, there was no change in the shape, site, or posture of the phantom, but the pain in it increased. In 1927, neuromata were excised from the scar; this operation improved the pain, caused the phantom to rotate inwards, and increased the appreciation of its site; but its shape and length relative to the shoulder remained unchanged at first, though very gradually during the next 15 years the limb shortened till now the phantom hand corresponds in site to the normal elbow. During the day the phantom was practically unobtrusive, though he could recall it by " thinking about it "; when he was tired, e.g. at the end of a day's work, the hot, swollen phantom reappeared. Thundery and wet weather always intensified the pain.

Five weeks before admission, after walking 300 yards up a steep incline, he noticed pain in his left shoulder and in the phantom hand "as though it had been battered to pulp"; this was followed when effort was continued by suffocation, constriction of the chest, pains in neck and jaws, and he felt " as though he would have died had he kept on walking." He rested and the pain eased. Since then, however, pain has recurred with gradually lessening exertion, until now walking 150 yards on the flat will bring on in unvarying sequence pain in the phantom, then in the neck, then a sense of constriction in the chest and choking. In the past fortnight there has been increasing breathlessness; now the act of undressing at bedtime leaves him breathless and exhausted and brings on the pain. He has had no cough, sputum, or other chest symptoms; no indigestion; no bladder disturbance; and no loss of weight.

Examination showed the scars of the disarticulation extending from the left acromio-clavicular

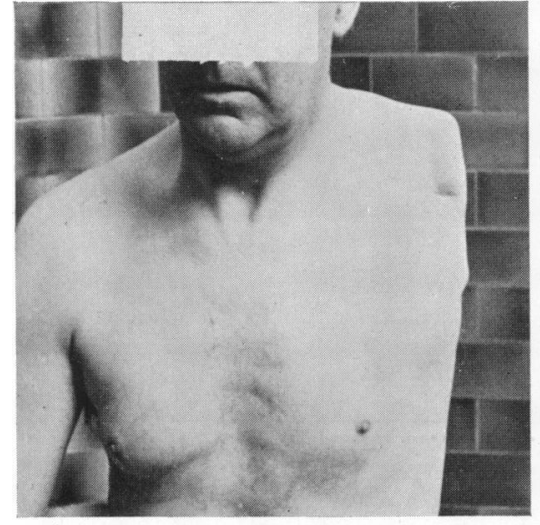

A

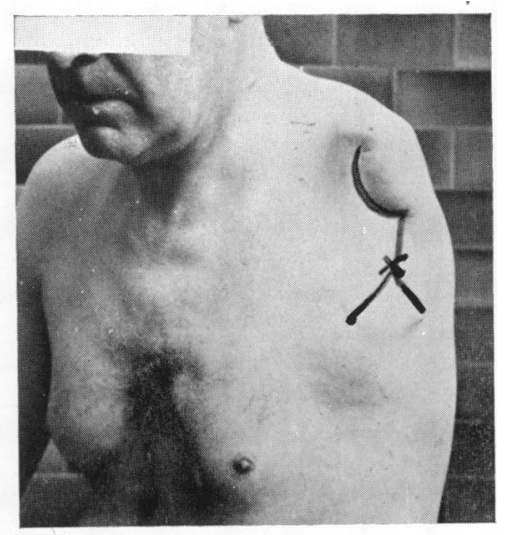

B

FIG. 3.-Case 2. (A) Showing site of the amputation through the shoulder joint.

(B) The scar is inked in and a pin-head neuroma indicated at X.

joint to the fifth rib in the mid-axillary line (Fig. 3); it was tender at $\mathrm{X}$ (Fig. 3B); pressure on this spot gave rise to a bursting sensation in the phantom similar to that experienced on exertion.

The heart was slightly enlarged and the X-ray report was: Slight enlargement of the left ventricle; bilateral simple fibrosis of the lungs. The sounds were normal, the rhythm regular and there was no murmur. B.P. 120/70. Pulse rate 84. Early locomotor brachial artery with slight silver wire thickening of retinal arteries. A cardiogram (Fig. 4B) showed low voltage and negative $T_{2}$ and $T_{3}$ waves.

$\mathrm{He}$ had slight emphysema, and an early arcus senilis. There was no organic lesion in the nervous system; no evidence of sepsis in mouth (edentulous), or naso-pharynx; no abdominal abnormality except the appendix scar which was well healed; no thyroid change; no rash; no palpable lymph nodes.

The urine was normal. The blood showed no anæmia. The Wassermann reaction was negative.

On June 5, 1942, Mr. Edwards blocked the left brachial plexus with 60 c.c. of 1 per cent solution of procaine. The diagram of the resulting anæsthesia (Fig. 5) shows that the lower part of the scar escaped, due to its dorsal nerve supply. On anæsthetization, the phantom lengthened and felt "like the gum when the dentist injects a local anæsthetic." No ocular or vascular changes were seen. About 20 minutes later he walked briskly on the flat (at a rate of 96 paces of two feet a minute): after 200 yards the suffocation and pain in the neck appeared but there was no pain in the hand; as he continued to walk he became conscious of the presence of the phantom and could discern the middle finger as a separate structure; and finally, when pain in the chest was so severe that he had to stop (after 600 yards), he was beginning to feel a mild bursting sensation in the phantom hand. 

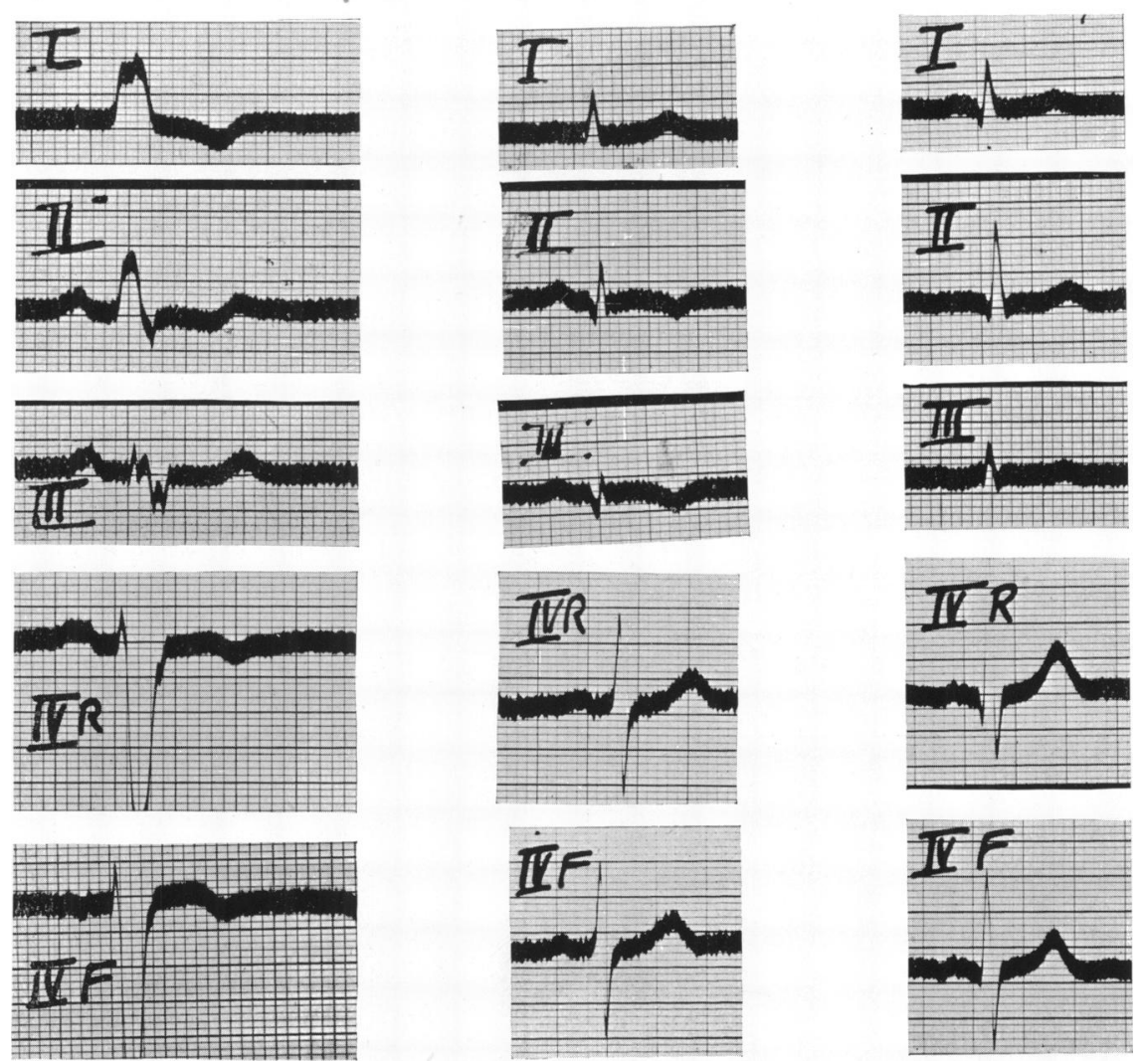

B

C

Fig. 4.-(A). Case I. Cardiogram showing left bundle branch block.

(B) Case 2. Cardiogram showing low voltage and negative $T$ in leads II and III.

(C) Cardiogram from control patient with left upper phantom but no cardiac pain.
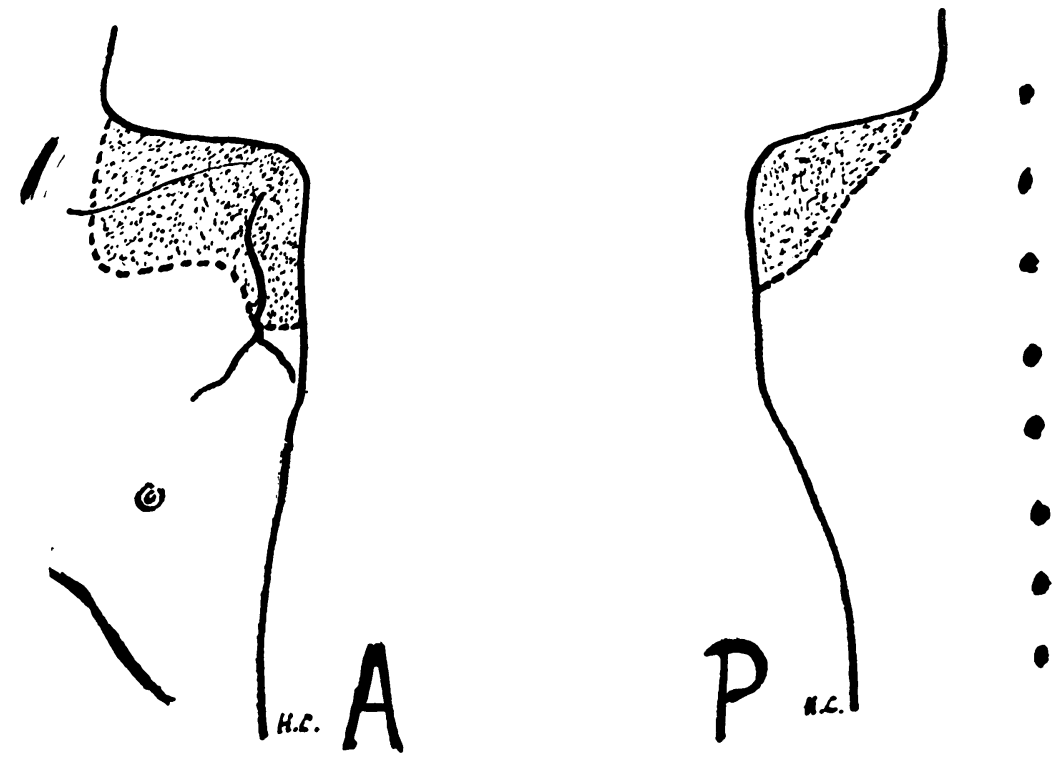

Fig. 5.--Case 2. The dotted area maps the anæsthesia resulting from brachial plexus block. A. Anterior view. P. Posterior view. 
When the effects of the anæsthetic passed, the sequence of the angina reverted to phantom first (which appeared after about $\mathbf{1 5 0}$ yards walking on the level at a rate of 96 paces of 2 feet a minute), followed by suffocation, chest constriction, and neck pain (after 200-250 yards). A week later normal saline was injected into the plexus; this did not modify in any way the distribution of the pain, the order of appearance of its components, or the intensity of effort needed to induce it.

On July 20, 4 c.c. of 2 per cent novocaine was injected into the region of the neuroma at X (Fig. 3B). The effect on the cardiac pain was similar in type to that following the plexus anæsthesia. After walking 220 yards, constriction of the chest and choking appeared; as he continued this became very severe and then he felt the bursting pain in the phantom hand.

\section{COMMENTS}

Fig. $4 \mathrm{C}$ was taken from a patient with a phantom left arm who was otherwise quite well. It shows that fixing the left arm electrode to the amputation stump in no way modifies the cardiogram.

Great care was taken to avoid infiltrating the stellate ganglion when injecting the brachial plexus, as it is known that angina can be relieved by blocking the afferent impulses arising from the heart and passing through this ganglion. Apart from the visible evidence of the extent of the plexus infiltration two facts showed that the stellate ganglion had not been anæsthetized : first, the absence of any ocular or vascular signs of sympathetic paralysis; and secondly, the persistence of anginal pain in the areas not rendered anæsthetic by the brachial plexus block; had the stellate ganglion been blocked all afferent impulses from the heart would have been prevented from reaching the sensorium.

A careful search of the available journals has failed to reveal any comparable record of a patient who referred anginal pain to a " phantom." It is proposed in a later paper to discuss the bearing of our cases and the results of brachial plexus and neuroma block on current theories of referred visceral pain. It will be patent, however, that no such theory is tenable which fails to account for (i) reference of the pain to a phantom, and (ii) the effects of brachial plexus and neuroma block.

\section{SUMMARY}

Two cases are recorded of cardiac pain referred to a phantom left arm.

Anæsthetization of the brachial plexus of the phantom caused, in one case, abolition of the phantom component of the cardiac pain, and in the other significant delay in the appearance of the phantom component which led to a reversal of the site of onset and spread of the cardiac pain. 